\title{
Maintenance chemotherapy after 6 cycles of platinum-doublet regimen in anthracycline-and taxane-pretreated metastatic breast cancer
}

\author{
Eun Kyo Joung ${ }^{1}$, Ji Hyun Yang ${ }^{2}$, Sooeun $\mathrm{Oh}^{3,4}$, Se Jun Park ${ }^{5}$, and Jieun Lee $\mathrm{Le}^{4,5}$
}

\begin{abstract}
${ }^{1}$ Department of Internal Medicine, College of Medicine, The Catholic University of Korea, Seoul; ${ }^{2}$ Division of Medical Oncology, Department of Internal Medicine, Uijeongbu St. Mary's Hospital, College of Medicine, The Catholic University of Korea, Uijeongbu; ${ }^{3}$ Department of Hospital Pathology, College of Medicine, The Catholic University of Korea, Seoul; ${ }^{4}$ Cancer Research Institute, The Catholic University of Korea, Seoul; ${ }^{5}$ Division of Medical Oncology, Department of Internal Medicine, Seoul St. Mary's Hospital, College of Medicine, The Catholic University of Korea, Seoul, Korea
\end{abstract}

Received: April 16, 2019

Revised : May 30, 2019

Accepted: July 26, 2019

\section{Correspondence to \\ Jieun Lee, M.D.}

Division of Medical Oncology,

Department of Internal Medicine, Seoul St. Mary's Hospital, College of Medicine, The Catholic University of Korea, 222

Banpo-daero, Seocho-gu, Seoul 06591, Korea

Tel: $+82-2-2258-6048$

Fax: +82-2-2258-6048

E-mail: befamiliar@catholic.ac.kr https://orcid.org/0000-0002$2656-0650$
Background/Aims: Sequential monotherapy is recommended for anthracyclineand taxane-resistant metastatic breast cancer (MBC), but combination chemotherapy is considered in patients with visceral crisis. Cisplatin-doublet chemotherapy is a combination regimen for $\mathrm{MBC}$, but prolonged treatment is challenging because of toxicity. We analyzed the role of single-agent maintenance chemotherapy after cisplatin-doublet chemotherapy for MBC.

Methods: From January 2011 to December 2017, 96 anthracycline- and taxane-resistant MBC patients were retrospectively reviewed, and 49 patients with a sustained clinical benefit during the initial 6 cycles of cisplatin-doublet chemotherapy were enrolled for study. Patients were treated with gemcitabine-cisplatin (gemcitabine, $1,250 \mathrm{mg} / \mathrm{m}^{2}$, intravenously [IV], days 1 to 8 ; cisplatin $60 \mathrm{mg} / \mathrm{m}^{2}$, IV, day 1 ) or capecitabine-cisplatin (capecitabine $2,500 \mathrm{mg} / \mathrm{m}^{2}$, orally, days 1 to 14 ; cisplatin $60 \mathrm{mg} / \mathrm{m}^{2}, \mathrm{IV}$, day $\mathrm{l}$ ) during the induction period. After 6 cycles, 16 patients were switched to single-maintenance treatment (gemcitabine or capecitabine) and the doublet regimen was continued in 24 patients. Survival outcomes (progression-free survival [PFS] and overall survival [OS]) were analyzed.

Results: Among the 49 patients who showed a clinical benefit during cisplatin-doublet therapy, 24 were maintained on the doublet regimen, 16 were switched to single-maintenance treatment, and chemotherapy was suspended until disease progression in nine patients. The single-maintenance chemotherapy group showed superior survival than the chemotherapy holiday and doublet regimen groups (median PFS 15.43 months vs. 8.37 and 10.67 months, respectively, $p=0.008$; median OS 43.67 months vs. 22.17 and 22.33 months, respectively, $p=0.014$ ).

Conclusions: Patients showing a clinical benefit during 6 cisplatin-doublet chemotherapy cycles may have a sustained survival benefit from single-maintenance chemotherapy.

Keywords: Breast neoplasms; Cisplatin; Maintenance chemotherapy

\section{INTRODUCTION}

Metastatic breast cancer (MBC) is considered an incurable disease [1]. For life prolongation and symptom pal- liation of MBC patients, sequential systemic treatment and supportive care is required. Recently, the survival duration of MBC patients has markedly improved and thus maintaining the quality of life during system- 
ic treatment is becoming more important [1]. Further, as pronged survival has made MBC a chronic disease among cancer patients, its management is an ongoing clinical challenge.

Chemotherapy is recommended in triple-negative breast cancer, luminal-type breast cancer with endocrine resistance, human epidermal growth factor 2 (HER-2)-positive breast cancer with anti-HER-2 treatment, and in patients presenting with visceral crisis. A standard chemotherapy sequence following anthracycline and taxane failure has not yet been established in MBC. Monotherapy is preferred during treatment, but for patients who show rapid progression, with massive tumor burden or symptomatic disease, combination chemotherapy can be considered [2]. Among diverse chemotherapeutic agents, cisplatin is one of the candidates for a combination regimen. Cisplatin has low activity as a single agent for previously-treated MBC, but combination with other chemotherapeutic agents appears to enhance its activity [3]. A cisplatin-doublet regimen showed clinical activity in triple-negative breast cancer [4], and clinical response was also proven in other subtypes of breast cancer [5-7]. Cisplatin can be combined with several other agents including vinorelbine, paclitaxel, docetaxel, gemcitabine, and capecitabine [3-9].

Among patients showing a clinical response during cisplatin-doublet treatment, most eventually show intolerance to cisplatin because of its cumulative toxicities, such peripheral neuropathy, hearing difficulties, electrolyte imbalance, delayed bone marrow recovery, and nausea. Considering its toxicities, some clinical trials were designed to administer cisplatin up to a maximum of 6 to 8 cycles $[4,10,11]$. At present, most clinicians determine the administration duration of cisplatin-doublet chemotherapy on the basis of their clinical experience. In patients showing clinical benefit during cisplatin-doublet treatment, there is no consensus about the role of prolonged doublet-maintenance chemotherapy or treatment holiday. There is a positive role of maintenance chemotherapy in breast cancer [12], but the toxicity of chemotherapy makes doublet-maintenance chemotherapy challenging. In the case of non-small cell lung cancer, the benefit of single-agent maintenance chemotherapy was established after induction platinum-doublet chemotherapy, according to the PARAMOUNT trial [13]. Single-agent maintenance chemotherapy after induction cisplatin-doublet chemotherapy can also be considered in breast cancer, but there are no randomized clinical trials for confirmation of the optimal duration of cisplatin-doublet induction treatment and the role of single-agent maintenance treatment thereafter.

To determine the appropriate duration of cisplatin-doublet administration and to verify the role of single-agent maintenance chemotherapy, the authors analyzed the clinical outcomes and toxicities for patients who were treated with cisplatin-doublet chemotherapy, that is, a gemcitabine-cisplatin (GP) or capecitabine-cisplatin (XP) regimen. The primary aim of the study was to determine the optimal duration of cisplatin-doublet chemotherapy and the role played by single-agent maintenance chemotherapy after induction cisplatin-doublet chemotherapy in MBC patients resistant to anthracycline and taxane.

\section{METHODS}

\section{Patients}

From January 2011 to December 2017, the medical records of patients who were diagnosed with recurrent or metastatic breast cancer at Seoul St. Mary's Hospital were retrospectively reviewed. Ninety-six patients treated with cisplatin-doublet chemotherapy (GP or $\mathrm{XP}$ ) after anthracycline and taxane treatment failure were selected. Among the selected patients, 49 patients showing sustained clinical response (complete response, partial response) or stable disease during the initial 6 cycles of cisplatin-doublet chemotherapy were enrolled for analysis. Other inclusion criteria were as follows: (1) pathologically proven invasive ductal or lobular carcinoma in surgical or biopsy specimens; (2) an Eastern Cooperative Oncology Group (ECOG) performance status of o to 2; (3) evaluable lesions based on Response Evaluation Criteria in Solid Tumors (RECIST) version 1.1; and (4) adequate bone marrow function, renal function, and hepatic function. This study was approved by the Institutional Review Board (IRB) of Seoul St. Mary's Hospital, Catholic University of Korea (KC17RESIo273). The requirement for written informed consent was waived according to the decision of IRB. 


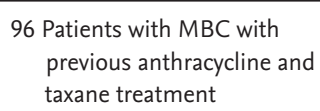

6 Cycles of cisplatin-doublet regimen
-GP or XP

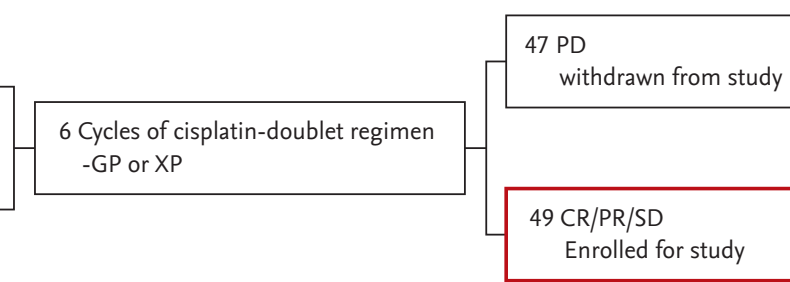

Enrolled for study
24 Doublet-maintenance

16 Single-maintenance

9 Chemotherapy holiday

\section{A}

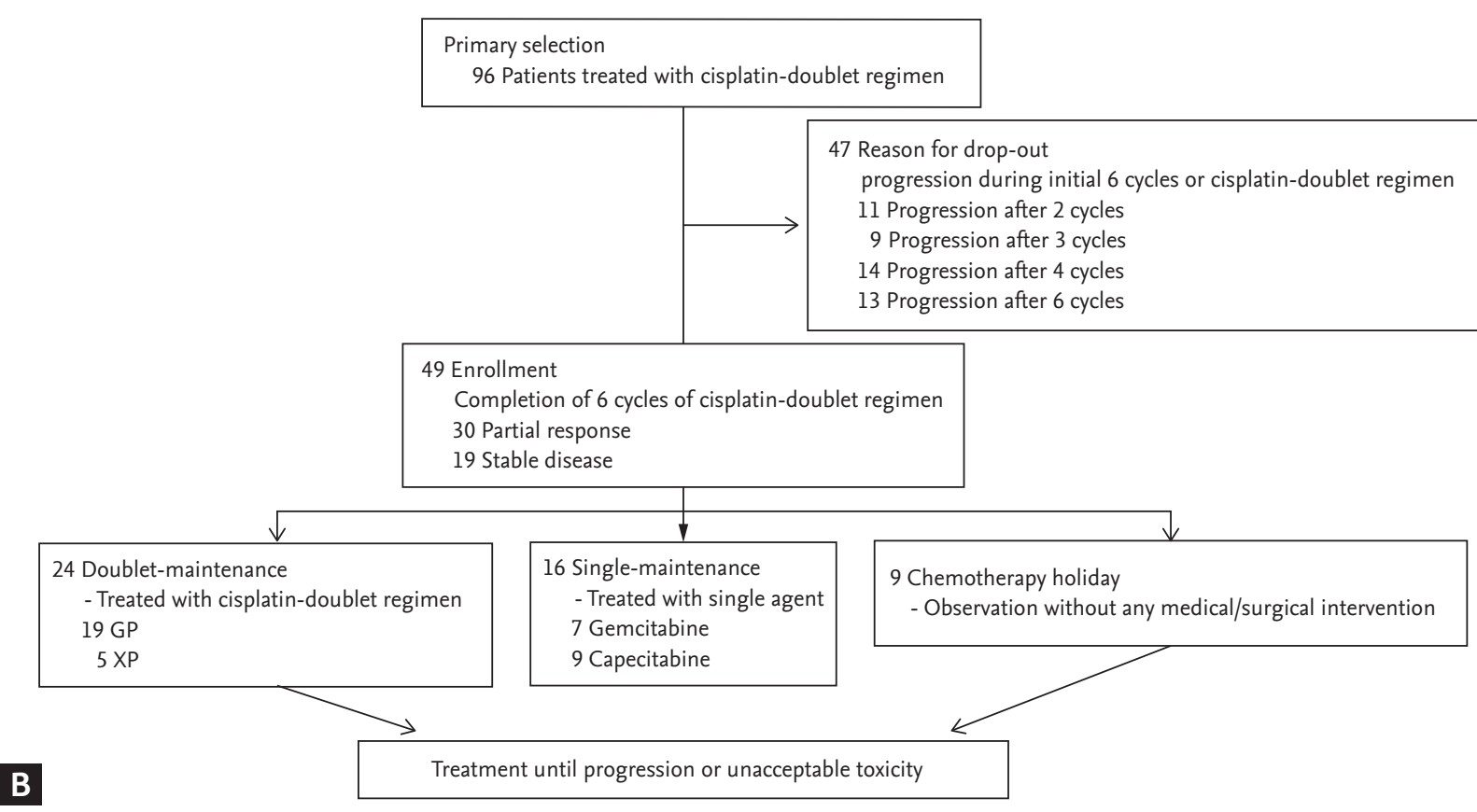

Figure 1. Treatment scheme (A) and consort diagram (B) of patients. MBC, metastatic breast cancer; GP, gemcitabine-cisplatin; $\mathrm{XP}$, capecitabine cisplatin; $\mathrm{PD}$, progressive disease; $\mathrm{CR}$, complete response; $\mathrm{PR}$, partial response; $\mathrm{SD}$, stable disease.

\section{Treatment schedule and response evaluation}

All patients were treated with a combination of either gemcitabine and cisplatin (GP: gemcitabine $1,250 \mathrm{mg} /$ $\mathrm{m}^{2}$, intravenous [IV], days 1 and 8; cisplatin $60 \mathrm{mg} / \mathrm{m}^{2}$, IV, day 1 ) or capecitabine and cisplatin (XP: capecitabine $1,000 \mathrm{mg} / \mathrm{m}^{2}$ twice a day, oral administration, 14 days of treatment followed by 7 days of rest; cisplatin $60 \mathrm{mg} / \mathrm{m}^{2}$, IV, day 1) every 3 weeks. One liter of half saline was delivered before and after cisplatin administration.

Response evaluation was performed using computed tomography (CT) scans obtained after every two cycles of chemotherapy, using RECIST criteria version 1.1. Toxicity was assessed based on National Cancer Institute Common Terminology Criteria for Adverse Events,version 4.0, during each cycle of chemotherapy. Chemotherapy was administered until progressive disease or unacceptable toxicity was observed.

\section{Definition of doublet-maintenance chemotherapy, single-maintenance chemotherapy, and chemotherapy holiday}

Patients were initially treated with a cisplatin-doublet regimen. Among the patient population, those who showed a sustained clinical response (complete response, partial response) or stable disease during the initial 6 cycles of cisplatin doublet chemotherapy were selected for analysis (Fig. 1A). We defined 6 cycles as the timepoint for minimal cisplatin administration, according to previous studies limiting the cisplatin-doublet regimen to up to 6 cycles considering the toxicity 
associated with cisplatin accumulation [10-12]. Patients who received persistent cisplatin-doublet chemotherapy for more than 6 cycles were defined as the doublet-maintenance group. Patients who received a single agent (gemcitabine or capecitabine) after 6 cycles of cisplatin-doublet were defined as the single-maintenance group. Patients in whom chemotherapy was suspended without any medical or surgical intervention after 6 cycles of doublet chemotherapy were defined as the chemotherapy holiday group. The doublet-maintenance and single-maintenance chemotherapy groups received cisplatin-doublet chemotherapy or single-maintenance chemotherapy, respectively, until progressive disease or unacceptable toxicity was observed (Fig. 1B).

\section{Statistical analysis}

Progression-free survival (PFS) was calculated from the first date of cisplatin-based doublet chemotherapy (XP or GP) to the date of disease progression, as shown by CT scans or patient death. Overall survival (OS) was calculated from the first date of cisplatin-based doublet chemotherapy to patient death or the last follow-up date. PFS after 6 cycles of cisplatin-doublet therapy was calculated from the first date of the 7 th cycle of chemotherapy to the date of disease progression, as shown by CT scans or patient death. OS after 6 cycles of cisplatin-doublet was calculated from the first date of the 7 th cycle of chemotherapy to patient death or last follow-up date. Response rate $(\mathrm{RR})$ was defined as the patient proportion showing complete response (CR) or partial response (PR) over total patient population, based on RECIST version 1.1. Disease control rate (DCR) was defined as the proportion of patients with $\mathrm{CR}, \mathrm{PR}$, or stable disease (SD) over the total patient population, based on RECIST version 1.1.

Continuous variables were presented as median values, and categorical variables were presented as percentages. Continuous variables were compared using the Kruskal-Wallis test, whereas categorical variables were compared using chi-square tests and Fisher's exact tests. Survival analyses were performed using the Kaplan-Meier method, and the results were compared using a log-rank test. The prognostic factors were analyzed using the multivariate Cox regression method, including those that were statistically significant in the univariate analysis. A p values $<0.05$ were considered statistically significant. The results were reported as odds ratios (OR) with 95\% confidence intervals (CI). SPSS version 24.0 for Windows (IBM Co., Armonk, NY, USA) was used for all statistical analyses in this study.

\section{RESULTS}

\section{Baseline patient characteristics}

From January 2011 to December 2017, 96 patients who were treated with a cisplatin-doublet regimen (GP, XP) were screened for eligibility. All patients were previously treated with anthracycline- and taxane-based chemotherapy (Supplementary Table 1). After 6 cycles of cisplatin-doublet chemotherapy, 49 patients who showed a clinical response or stable disease during treatment were enrolled for study. The baseline patient characteristics are described in Table 1 . Among the total patient population, 24 patients (48.9\%) received continued cisplatin-doublet maintenance (doublet-maintenance group), 16 patients $(32.6 \%)$ received single-agent maintenance after 6 cycles of cisplatin-doublet therapy (single-maintenance group), and chemotherapy was suspended after 6 cycles of cisplatin-doublet therapy in nine patients (18.5\%) (chemotherapy holiday group) (Supplementary Table 1). The median age of the patients was 53 years in the doublet-maintenance group and 48 years in the single-maintenance group. There were relatively fewer triple-negative breast cancer patients in the single-maintenance group (three patients, 18.8\%) compared with the doublet-maintenance group (six patients, $25 \%$ ), but there were no statistically significant differences between the two groups $(p=0.717)$.

\section{Administration of doublet- and maintenance- chemotherapy}

Among the continuation-treatment groups, a median of three cycles of cisplatin-doublet therapy was delivered to the doublet-maintenance group, and a median of 10 cycles of a single-maintenance regimen was delivered to the single-maintenance group. In the doublet-maintenance group, gemcitabine was the preferred combination regimen with cisplatin (19 patients, 79.2\%). However, in the single-maintenance chemotherapy group, more patients received capecitabine maintenance (nine patients, $56.3 \%$ ) as compared with gemcitabine maintenance (seven patients, 43.8\%) (Table 2). 
Table 1. Baseline patient characteristics

\begin{tabular}{|c|c|c|c|}
\hline Characteristic & Doublet-maintenance $(\mathrm{n}=24)$ & Single-maintenance $(n=16)$ & $p$ value \\
\hline Age, yr & $53(39-69)$ & $49(30-70)$ & 1.000 \\
\hline$<65$ & $23(95.8)$ & $15(93.8)$ & \\
\hline$\geq 65$ & $1(4.2)$ & $1(6.3)$ & \\
\hline ECOG & & & 0.262 \\
\hline $0-1$ & $21(87.5)$ & $16(100.0)$ & \\
\hline 2 & $3(12.5)$ & o & \\
\hline Hormone receptor status & & & 0.717 \\
\hline Positive & $18(75.0)$ & $13(81.2)$ & \\
\hline Negative & $6(25.0)$ & $3(18.8)$ & \\
\hline HER2 positive & $4(16.7)$ & $3(18.8)$ & 1.000 \\
\hline Triple negative & $6(25.0)$ & $3(18.8)$ & 0.717 \\
\hline Disease status & & & 0.439 \\
\hline Metastatic breast cancer & $18(75.0)$ & $14(87.5)$ & \\
\hline Recurrent breast cancer & $6(25.0)$ & $2(12.5)$ & \\
\hline \multicolumn{4}{|l|}{ Neoadjuvant chemotherapy } \\
\hline Anthracycline & $2(8.3)$ & $2(12.5)$ & 1.000 \\
\hline Taxane & $2(8.3)$ & 0 & 0.496 \\
\hline \multicolumn{4}{|l|}{ Adjuvant chemotherapy } \\
\hline Anthracycline & $11(45.8)$ & $9(56.3)$ & 1.000 \\
\hline Taxane & $5(20.8)$ & $5(31.3)$ & 0.707 \\
\hline Previous lines of palliative chemotherapy & & & 0.360 \\
\hline Naïve & $1(4.2)$ & $1(6.3)$ & \\
\hline 1st & $6(25.0)$ & $6(37.5)$ & \\
\hline 2nd & $6(25.0)$ & $4(25.0)$ & \\
\hline 3 rd & $3(12.5)$ & $3(18.8)$ & \\
\hline$\geq 4$ th & $8(33.3)$ & $2(12.5)$ & \\
\hline \multicolumn{4}{|l|}{ Previous chemotherapy agents } \\
\hline Gemcitabine & $4(16.7)$ & $1(6.3)$ & 0.631 \\
\hline Vinorelbine & $8(33 \cdot 3)$ & $3(18.8)$ & 0.473 \\
\hline CMF & $5(20.8)$ & $3(18.8)$ & 1.000 \\
\hline Capecitabine & $18(75.0)$ & $10(62.5)$ & 0.272 \\
\hline Previous palliative endocrine treatment & $8(33 \cdot 3)$ & $6(37.5)$ & 1.000 \\
\hline Previous palliative HER2 treatment & $5(20.8)$ & $1(6.3)$ & 0.373 \\
\hline No. of metastatic sites & & & 0.236 \\
\hline 1 & $6(25.0)$ & $3(18.8)$ & \\
\hline 2 & $7(29.2)$ & $10(62.5)$ & \\
\hline$\geq 3$ & $11(45.8)$ & $3(18.8)$ & \\
\hline \multicolumn{4}{|l|}{ Metastatic sites } \\
\hline Distant lymph nodes & $13(54.2)$ & $3(18.8)$ & 0.047 \\
\hline Lung & $9(37.5)$ & $8(50.0)$ & 0.433 \\
\hline Liver & $8(33.3)$ & $7(43.8)$ & 0.505 \\
\hline Bone & $11(45.8)$ & $8(50.0)$ & 0.796 \\
\hline Pleura & $6(25.0)$ & $3(18.8)$ & 0.717 \\
\hline Brain & $5(20.8)$ & $3(18.8)$ & 1.000 \\
\hline Visceral metastases & $18(75.0)$ & $15(93.8)$ & 0.210 \\
\hline
\end{tabular}

Values are presented as median (range) or number (\%).

ECOG, Eastern Cooperative Oncology Group; HER2, human epidermal growth factor 2; CMF, cyclophosphamide (oral) + methotrexate + -fluorouracil. 
Table 2. Administered chemotherapy agents and relative dose intensity

\begin{tabular}{|c|c|c|c|}
\hline Variable & Doublet-maintenance $(n=24)$ & Single-maintenance $(n=16)$ & $p$ value \\
\hline Total cycle after 6 cycles of cisplatin & $3(2-9)$ & $10(2-51)$ & 0.001 \\
\hline Combination agent & & & 0.022 \\
\hline Gemcitabine & $19(79.2)$ & $7(43.8)$ & \\
\hline Capecitabine & $5(20.8)$ & $9(56.3)$ & \\
\hline \multicolumn{4}{|l|}{ Relative dose intensity } \\
\hline Cisplatin & $78.0(60.0-100.0)$ & - & \\
\hline Gemcitabine & $79.0(66.7-100.0)$ & $73.3(60.0-80.0)$ & \\
\hline Capecitabine & $80(70-85)$ & $80.0(80.0-100.0)$ & \\
\hline
\end{tabular}

Values are presented as median (range) or number (\%).

Table 3. Clinical outcomes during maintenance treatment

\begin{tabular}{|c|c|c|c|c|c|c|}
\hline & \multicolumn{3}{|c|}{ Doublet-maintenance $(n=24)$} & \multicolumn{3}{|c|}{ Single-maintenance $(n=16)$} \\
\hline & GP & $\mathrm{XP}$ & Total & Gemcitabine & Capecitabine & Total \\
\hline Maintenance of response & $8(33 \cdot 3)$ & $2(8.4)$ & $10(41.7)$ & $6(37.5)$ & $9(56.3)$ & $15(93.8)$ \\
\hline Progression & $11(45.8)$ & $3(12.5)$ & $14(58.3)$ & $1(6.2)$ & 0 & $1(6.2)$ \\
\hline
\end{tabular}

Values are presented as number (\%).

GP, gemcitabine cisplatin; XP, capecitabine cisplatin.

The median relative dose intensity of cisplatin in the doublet-maintenance group was $77.8 \%$ (range, $66.7 \%$ to $90 \%$ ). In the single-maintenance group, the median relative dose intensity of gemcitabine was slightly lower (73.3\%) as compared to that in the doublet-maintenance group (79\%), but there was no difference in the relative dose intensity of capecitabine (Table 2).

\section{Survival and treatment response in total patient population}

The median progression-free survival (PFS) and overall survival (OS) in the primary patient population $(96$ patients) was 5.5 months (range, 0.83 to 55.1 ) and 20.8 months (range, 3.03 to 73.3 ), respectively. During the initial 6 cycles of cisplatin-doublet chemotherapy, there were no statistically significant differences in PFS between the gemcitabine and capecitabine combinations (median 5.13 months vs. 4.23 months, respectively; $p=0.108$ ). The overall response rate and disease control rate during the initial 6 cycles of cisplatin-doublet therapy were $37.5 \%$ and $81.3 \%$, respectively (Supplementary Table 2).

\section{Treatment response and survival outcomes according to duration of cisplatin administration, single-agent maintenance, or chemotherapy holiday} The single-maintenance chemotherapy group showed superior PFS than the chemotherapy holiday and doublet-maintenance chemotherapy groups (median 15.43 months, compared to 8.37 and 10.67 months, respectively; $p=0.008$ ) (Fig. 2A). The results for OS were similar, with superior OS in the single-maintenance group than in the chemotherapy holiday and doublet-maintenance chemotherapy groups (median 43.67 months compared to 22.17 and 22.33 months, respectively; $p=0.014$ ) (Fig. 2B). The single-maintenance chemotherapy group still showed superior PFS and OS after 6 cycles of cisplatin-doublet treatment compared to the doublet-maintenance or chemotherapy holiday groups ([median PFS after 6 cycles of cisplatin 9.57 months vs. 5.70 and 3.37 months, respectively; $p=0.005$ ], [median OS after six cycles of cisplatin 37.08 months vs. 17.90 and 17.07 months], respectively; $p=0.007$ ) (Fig. 3). Among the continuation-treatment groups, 15 patients (93.7\%) in the single-maintenance group maintained prior clinical response during follow-up. However, relatively fewer 

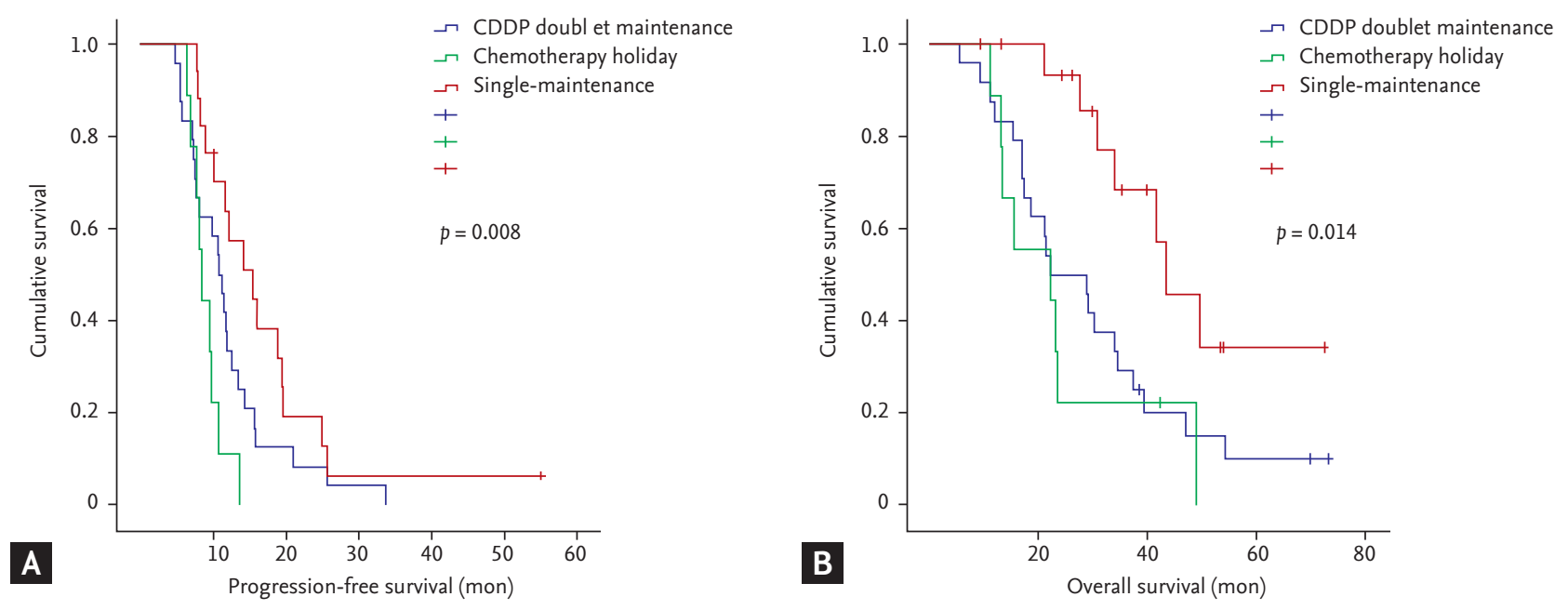

Figure 2. Survival outcomes of the patients who showed clinical response or stable disease after 6 cycles of cisplatin (CDDP)-doublet chemotherapy. (A) Progression-free survival. (B) Overall survival.
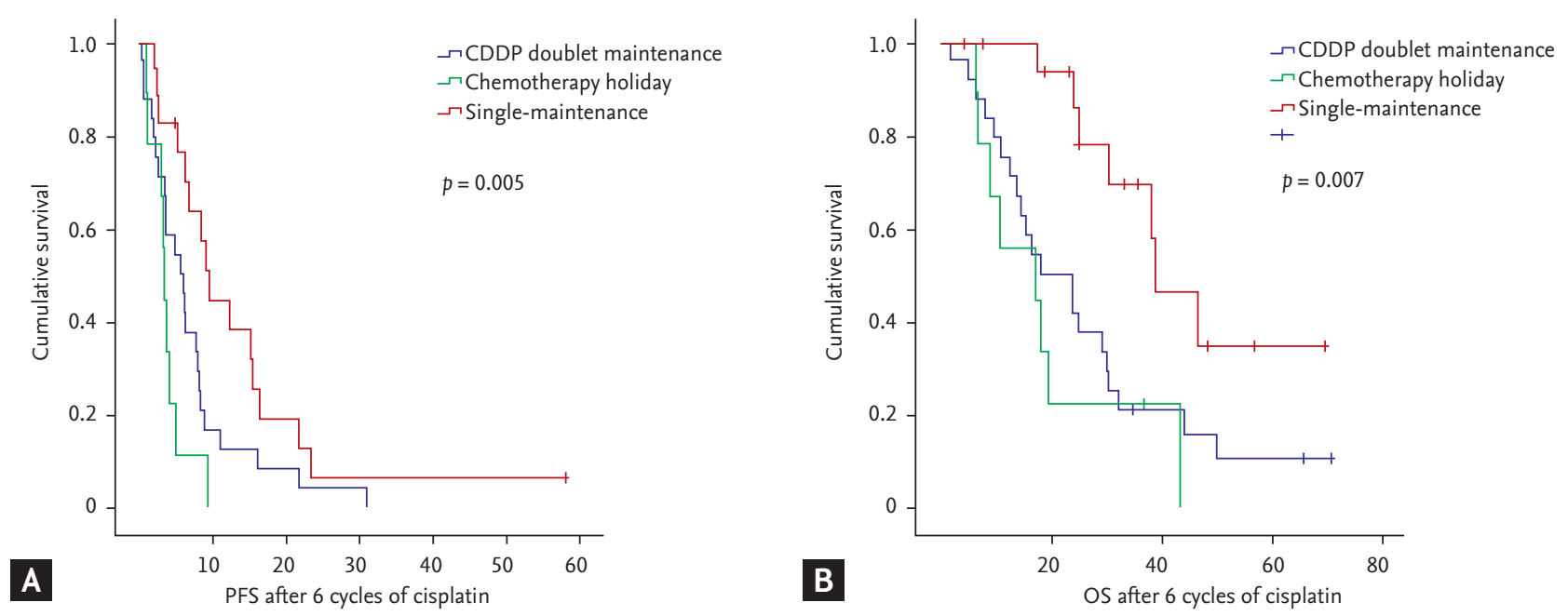

Figure 3. Survival outcomes after 6 cycles of cisplatin (CDDP)-doublet chemotherapy. (A) Progression-free survival (PFS). (B) Overall survival (OS).
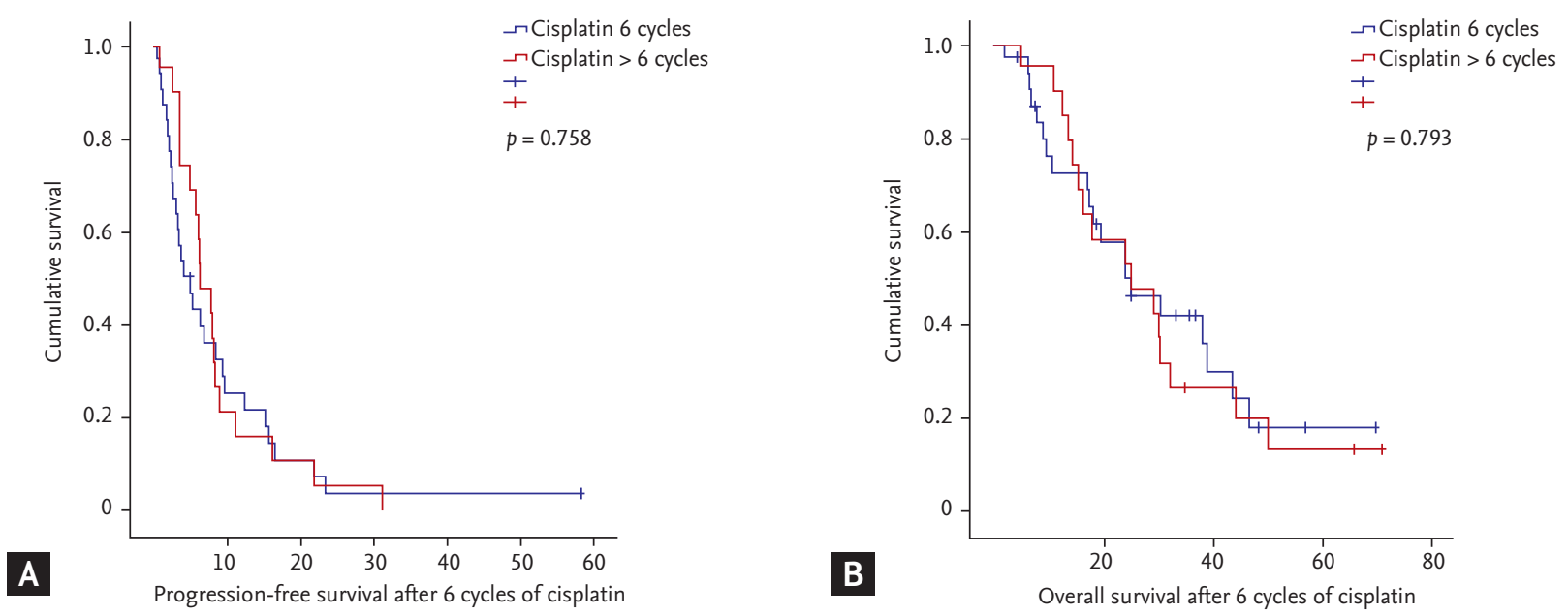

Figure 4. Survival outcomes of patients according to number of cisplatin-doublet administration. (A) Progression-free survival. (B) Overall survival. 
Table 4. Univariate and multivariate analysis of survival outcomes in doublet or single- maintenance group

\begin{tabular}{|c|c|c|c|c|c|c|c|c|}
\hline \multirow{3}{*}{ Characteristic } & \multicolumn{4}{|c|}{ Progression free survival } & \multicolumn{4}{|c|}{ Overall survival } \\
\hline & \multicolumn{2}{|c|}{ Univariate analysis } & \multicolumn{2}{|c|}{ Multivariate analysis } & \multicolumn{2}{|c|}{ Univariate analysis } & \multicolumn{2}{|c|}{ Multivariate analysis } \\
\hline & $\operatorname{HR}(95 \% \mathrm{CI})$ & pvalue & $\mathrm{HR}(95 \% \mathrm{CI})$ & $p$ value & $\mathrm{HR}(95 \% \mathrm{CI})$ & $p$ value & $\mathrm{HR}(95 \% \mathrm{CI})$ & $p$ value \\
\hline \multicolumn{9}{|l|}{ Age } \\
\hline$\leq 65$ vs. $>65$ & $3.07(0.92-10.19)$ & 0.067 & & & $1.44(0.34-6.16)$ & 0.622 & & \\
\hline \multicolumn{9}{|l|}{ ECOG } \\
\hline $0-1$ vs. 2 & $0.91(0.32-2.61)$ & 0.865 & & & $1.81(0.55-5.98)$ & 0.333 & & \\
\hline \multicolumn{9}{|l|}{ Subtype } \\
\hline HR positive vs. HER2 positive & $1.12(0.52-2.39)$ & 0.778 & $1.33(0.50-3.52)$ & 0.729 & $0.97(0.39-2.39)$ & 0.943 & $1.35(0.41-4.43)$ & 0.624 \\
\hline HR positive vs. TNBC & $1.56(0.75-3.28)$ & 0.237 & $1.78(0.79-4.02)$ & 0.422 & $1.94(0.84-4.49)$ & 0.121 & $2.04(0.79-5.24)$ & 0.138 \\
\hline \multicolumn{9}{|l|}{ Visceral metastasis } \\
\hline No vs. Yes & $1.34(0.56-3.19)$ & 0.506 & $1.08(0.44-2.66)$ & 0.874 & $0.48(0.21-1.09)$ & 0.082 & $1.43(0.54-3.79)$ & 0.476 \\
\hline \multicolumn{9}{|l|}{ Chemotherapy regimen } \\
\hline GP vs. XP & $1.87(0.98-3.56)$ & 0.056 & & & $2.14(0.99-4.58)$ & 0.051 & & \\
\hline \multicolumn{9}{|l|}{ Maintenance treatment } \\
\hline Doublet vs. Single & $0.51(0.26-0.99)$ & 0.047 & $0.49(0.24-1.00)$ & 0.049 & $0.36(0.15-0.86)$ & 0.021 & $0.37(0.15-0.95)$ & 0.038 \\
\hline
\end{tabular}

HR, hazard ratio; CI, confidence interval; ECOG, Eastern Cooperative Oncology Group; HR, hormone receptor; HER2, human epidermal growth factor 2; TNBC, triple negative breast cancer; GP, gemcitabine cisplatin; XP, capecitabine cisplatin.

Table 5. Chemotherapy toxicities

\begin{tabular}{|c|c|c|c|c|c|c|}
\hline \multirow{3}{*}{ Variable } & \multicolumn{2}{|c|}{$\begin{array}{c}\text { Initial } 6 \text { cycles of } \\
\text { cisplatin-doublet }(n=96)\end{array}$} & \multicolumn{4}{|c|}{ Continuation-treatment } \\
\hline & \multirow{2}{*}{ Any } & \multirow{2}{*}{ Grade 3-4 } & \multicolumn{2}{|c|}{ Doublet-maintenance $(n=24)$} & \multicolumn{2}{|c|}{ Single-maintenance $(n=16)$} \\
\hline & & & Any & Grade 3-4 & Any & Grade $3-4$ \\
\hline \multicolumn{7}{|l|}{ Hematologic } \\
\hline Anemia & $86(89.6)$ & $7(7 \cdot 3)$ & $13(54.2)$ & $4(16.7)$ & $14(87.5)$ & $2(12.5)$ \\
\hline Neutropenia & $79(82.3)$ & $52(54.2)$ & $10(41.7)$ & $5(20.8)$ & $10(62.5)$ & $2(12.5)$ \\
\hline Thrombocytopenia & $60(62.5)$ & $14(14.6)$ & $7(29.1)$ & $1(4.1)$ & $10(62.5)$ & $1(6.3)$ \\
\hline \multicolumn{7}{|l|}{ Non-hematologic } \\
\hline Neuropathy & $37(38.5)$ & $17(17.7)$ & $3(12.5)$ & 0 & $5(31.3)$ & o \\
\hline Nausea & $9(9.4)$ & $1(1)$ & $1(4.1)$ & o & 0 & o \\
\hline Vomiting & $5(5.2)$ & $1(1)$ & $1(4.1)$ & o & o & o \\
\hline Asthenia & $13(13.5)$ & 0 & 0 & o & o & o \\
\hline Stomatitis & o & 0 & 0 & o & 0 & 0 \\
\hline Diarrhea & $1(1)$ & o & $1(4.1)$ & o & o & o \\
\hline Hand-foot syndrome & $1(1)$ & $1(1)$ & o & 0 & 0 & 0 \\
\hline
\end{tabular}

Values are presented as number (\%).

patients (10 patients, 41.6\%) maintained prior clinical response in the doublet-maintenance group (Table 3).

In addition to treatment regimen, patients were subgrouped according to number of cisplatin administration cycles. There were no statistically significant differences in PFS (median 4.10 months vs. 6.27 months, $p=$ 0.758 ) or OS (median 23.90 months vs. 24.97 months, $p=$
0.793 ) according to 6 cycles of cisplatin-doublet administration (Fig. 4).

In the continuation-treatment groups, COX regression was performed for an in-depth analysis of the relationship between clinicopathologic parameters, administration of maintenance chemotherapy, and survival outcomes. Administration of single-maintenance 
Table 6. Systemic treatment after progression

\begin{tabular}{|c|c|c|c|}
\hline Variable & Doublet-maintenance $(n=24)$ & Single-maintenance $(n=16)$ & $p$ value \\
\hline Subsequent chemotherapy regimens & & & 0.433 \\
\hline Total & 41 & 30 & \\
\hline 1 & 18 & 11 & \\
\hline 2 & 14 & 10 & \\
\hline$\geq 3$ & 9 & 9 & \\
\hline Median & 2 & 3 & \\
\hline No. of total cycles & 196 & 205 & \\
\hline \multicolumn{4}{|l|}{ Type of chemotherapy regimens } \\
\hline Capecitabine & 7 & 4 & \\
\hline Gemcitabine & 3 & 5 & \\
\hline Eribulin & 10 & 8 & \\
\hline Vinorelbine & 6 & 1 & \\
\hline T-DMı & 1 & 1 & \\
\hline Paclitaxel & 2 & 2 & \\
\hline Docetaxel & 2 & 4 & \\
\hline Nab-paclitaxel & & 1 & \\
\hline CMF & 10 & 6 & \\
\hline Subsequent endocrine regimens & & & 0.441 \\
\hline Total & 21 & 19 & \\
\hline 1 & 13 & 9 & \\
\hline 2 & 7 & 6 & \\
\hline$\geq 3$ & 1 & 4 & \\
\hline \multicolumn{4}{|l|}{ Type of endocrine therapy } \\
\hline Tamoxifen & 3 & 2 & \\
\hline Letrozole & 6 & 6 & \\
\hline Exemestane & 4 & 3 & \\
\hline Exemestane + everolimus & 4 & 4 & \\
\hline Fulvestrant & 4 & 6 & \\
\hline
\end{tabular}

T-DM1, trastuzumab emtansine; CMF, cyclophosphamide (oral) + methotrexate + 5-fluorouracil.

chemotherapy was associated with superior PFS and OS compared with doublet-maintenance ([for PFS, hazard ratio [HR], 0.51; 95\% confidence interval [CI], 0.26 to $0.99 ; p=0.047$ ], [for OS, HR, $0.36 ; 95 \%$ CI, 0.15 to $0.86 ; p$ $=0.021])$. The type of chemotherapy regimen showed a borderline association with PFS and OS ([for PFS, HR, 1.87; 95\% CI, 0.98 to 3.56; $p=0.056$ ], [for OS, HR, 2.14; $95 \% \mathrm{CI}, 0.99$ to $4.58 ; p=0.051])$. When adjusted for tumor subtypes and the presence of visceral metastasis, administration of single-maintenance chemotherapy still showed a statistical association with prolongation of PFS and OS ([for PFS, HR, 0.49; 95\% CI, 0.24 to 1.00; $p$ $=0.049$ ], [for OS, HR, $0.37 ; 95 \% \mathrm{CI}, 0.15$ to $0.95 ; p=0.038]$ ]) (Table 4).

\section{Safety}

A total of 473 cycles of chemotherapy were administered in the total patient population. In the single-maintenance group, 225 cycles of chemotherapy were delivered after omission of cisplatin. In the doublet-maintenance group, 92 cycles of chemotherapy were delivered after the initial 6 cycles of cisplatin. There were no treatment-related deaths during chemotherapy. The hematologic and non-hematologic toxicities are summarized in Table 5. During the initial 6 cycles of chemotherapy, the most common grade 3 to 4 non-hematologic toxicity was peripheral neuropathy (17 patients, 17.7\%), and the most common grade 3 to 4 hematologic toxicity was neutropenia (52 patients, 54.2\%). Among the con- 
tinuation-treatment groups, any grade of peripheral neuropathy was slightly more frequently reported in the single-maintenance group (five patients, 31.3\%) as compared with the doublet-maintenance group (three patients, 12.5\%). However, the rates of gastrointestinal toxicities were higher in patients with doublet-maintenance chemotherapy (three patients, 21.3\%) than in patient with single-maintenance chemotherapy. In the single-maintenance group, the severity of peripheral neuropathy gradually decreased after the omission of cisplatin. Grade 3 to 4 hematologic toxicities were more frequently reported in the doublet-maintenance group.

\section{Systemic treatment after progression}

Details of systemic treatment after progression are listed in Table 6 . Thirty-five patients (71.4\%) received systemic chemotherapy after progression. One hundred ninety-six cycles of chemotherapy were administered to the doublet-maintenance group, and 205 cycles of chemotherapy were administered to the single-maintenance group. Cisplatin was not administered to any patients after disease progression. Among hormone receptor-positive breast cancer patients, $77.4 \%$ patients received hormonal treatment after disease progression. Details of systemic treatment after disease progression in the chemotherapy holiday group are listed in Supplementary Table 3.

\section{DISCUSSION}

In metastatic breast cancer (MBC), systemic treatment is designated according to pathologic subtypes. The mainstay of treatment in hormone receptor-positive breast cancer is based on endocrine treatment and cyclin-dependent kinase $4 / 6\left(\mathrm{CDK}_{4} / 6\right)$ inhibitors [1]. In the case of human epidermal growth factor receptor-2 (HER2)-positive breast cancer, anti-HER2 treatments such as trastuzumab and pertuzumab are the backbone of systemic therapy [14]. Eventually, however, all patients receive cytotoxic systemic chemotherapy in the event of visceral crisis, resistance to hormonal treatment, or resistance to anti-HER2 treatment. Guidelines suggest the use of systemic monotherapy after anthracycline and taxane failure. However, under certain circumstances, combination chemotherapy can be considered [2]. If pa- tients show a clinical benefit during combination chemotherapy, chemotherapy is generally maintained until disease progression; however, patients can suffer from complications of cytotoxic chemotherapy during prolonged treatment. To maximize the treatment effect and minimize the toxicity of chemotherapy during treatment, the concept of maintenance chemotherapy is introduced in various solid cancers, and this strategy has been well-established in non-small cell lung cancer (NSCLC) [15]. After four cycles of cisplatin-doublet chemotherapy, continuation maintenance chemotherapy with pemetrexed is the standard for NSCLC [13]. In the case of $\mathrm{MBC}$, maintenance chemotherapy has definite clinical benefits [12], but there are no established concepts of continuation maintenance with single chemotherapy agents to minimize chemotherapy-induced toxicities.

In this study, we evaluated the role of single-maintenance chemotherapy after cisplatin-doublet chemotherapy in anthracycline- and taxane-pretreated MBC patients. Among patients who showed clinical benefit to cisplatin-doublet treatment, single-maintenance chemotherapy showed the greatest survival benefit compared with doublet-maintenance or chemotherapy holiday. When considering the duration of cisplatin administration, there was no survival benefit in patients who received more than 6 cycles of cisplatin-doublet combination therapy. On the basis of our analysis, we hypothesized that maintenance chemotherapy after induction with cisplatin doublet chemotherapy may have a role in the treatment of MBC. Furthermore, our study provides a rationale for not administering cisplatin for more than 6 cycles when designing cisplatin-based chemotherapy in breast cancer. Until now, there have not been any guidelines for the duration of cisplatin administration during chemotherapy in breast cancer. Administration cycles of cisplatin-doublet therapy were variable among previous clinical trials, ranging from 6 cycles to maintenance of therapy until unacceptable toxicity $[6,10,16,17]$. Considering the results of our analysis, we can rationalize the use of cisplatin for a certain amount of cycles, followed by a transition into maintenance chemotherapy with a less toxic single agent to improve survival outcomes. In our analysis, all patients received at least 6 cycles of cisplatin-doublet treatment with clinical benefit. On the basis of other solid malignancies, such as NSCLC [13], four cycles of the cisplatin-doublet treatment and continuation 
of maintenance treatment after clinical response can also be considered, but further investigations through clinical trials are needed.

The overall response rate of cisplatin-doublet therapy in our analysis was $37.5 \%$ of the prescreened patient population, which was relatively lower than that recently reported in the literature, wherein cisplatin-based chemotherapy was administered at earlier timepoints $[4,5,10]$, but was comparable to that in other reports in which cisplatin was administered in heavily pre-treated $\mathrm{MBC}$ patients $[6,16]$. In our analysis, all patients were anthracycline- and taxane-resistant, and about $40 \%$ of the patient population received more than three lines of palliative chemotherapy before enrollment in the study. Furthermore, $42.7 \%$ of patients had more than three sites of metastasis, and about $80 \%$ of patients had visceral metastases. As patients in our analysis were heavily pretreated and had significant disease burden, cisplatin-doublet chemotherapy may have a role as palliative treatment in patients with MBC. Among patients who showed a beneficial response during cisplatin-doublet chemotherapy and received at least 6 cycles of cisplatin-doublet chemotherapy, our analysis showed a survival benefit in patients receiving single-maintenance chemotherapy, along with tolerable toxicity profiles. On the basis of this analysis, we can conclude that cisplatin-doublet chemotherapy may have a benefit in anthracycline- and taxane-resistant patients with relatively heavy disease burden. Among patients who show clinical response during cisplatin-doublet treatment, single-maintenance chemotherapy may lead to long-term disease control with favorably less toxicity.

There are some limitations that prompt careful interpretation of the study results. This study was a retrospective study, with a small sample size; thus the study results require careful interpretation. Systemic monotherapy is considered the standard therapy in treatment of $\mathrm{MBC}$, and relatively small portions of the patient population received combination chemotherapy during analysis. As cisplatin-doublet regimen is considered to be one of the treatment options of combination chemotherapy and is not established as the standard doublet regimen, only a small number of patients were eligible for the study. The rarity of the cisplatin-doublet regimen has influenced the hetero- geneous distribution of single-maintenance chemotherapy within the study. Furthermore, the time point and rationale for interrupting the administration of cisplatin were variable during the study. The heterogeneous patient population may have influenced the results by selection bias. However, our study holds its clinical value by suggesting the optimal duration of the cisplatin-doublet regimen to maximize survival outcomes and minimize the toxicity of the chemotherapy regimen. Although the cisplatin-doublet regimen is not the mainstay for treatment of MBC, there is a proportion of patients that does receive the cisplatin-doublet regimen during treatment. Presently, clinicians usually suggest a maximal tolerance of the cisplatin administration cycle on the basis of their clinical experience. Although our study is based on a retrospective analysis, our analysis suggests that 6 cycles of the cisplatin-doublet regimen maximize the clinical outcome. Furthermore, we have also shown that single-maintenance chemotherapy after cisplatin-doublet regimen may have a significant clinical benefit. Until now, there have been relatively few studies evaluating the role of maintenance chemotherapy in MBC. On the basis of this study, we are planning a prospective trial to evaluate the role of single-maintenance chemotherapy after six cycles of cisplatin-doublet chemotherapy. Furthermore, we can also consider shortening the duration of cisplatin-doublet regimen in patients with good clinical response in the future, with detailed clinical trials being warranted for complete understanding of the clinical outcomes.

\section{KEY MESSAGE}

1. Single-maintenance chemotherapy after a minimum of six cycles of cisplatin-doublet chemotherapy may have survival benefits, while minimizing chemotherapy-related toxicity in patients who show response during cisplatin-doublet chemotherapy.

2. Cisplatin-based chemotherapy, such as gemcitabine-cisplatin or capecitabine-cisplatin, may have a role in the treatment of pretreated metastatic breast cancer, particularly in patients with heavy disease burden. 


\section{Conflict of interest}

No potential conflict of interest relevant to this article was reported.

\section{REFERENCES}

1. Harbeck N, Gnant M. Breast cancer. Lancet 2017389:11341150.

2. Cardoso F, Senkus E, Costa A, et al. 4th ESO-ESMO international consensus guidelines for advanced breast cancer (ABC 4). Ann Oncol 2018;29:1634-1657.

3. Martin M. Platinum compounds in the treatment of advanced breast cancer. Clin Breast Cancer 2001;2:190-208.

4. $\mathrm{Hu} \mathrm{XC}$, Zhang J, Xu BH, et al. Cisplatin plus gemcitabine versus paclitaxel plus gemcitabine as first-line therapy for metastatic triple-negative breast cancer (CBCSGoo6): a randomised, open-label, multicentre, phase 3 trial. Lancet Oncol 2015;16:436-446.

5. Stemmler HJ, diGioia D, Freier W, et al. Randomised phase II trial of gemcitabine plus vinorelbine vs gemcitabine plus cisplatin vs gemcitabine plus capecitabine in patients with pretreated metastatic breast cancer. $\mathrm{Br} \mathrm{J}$ Cancer 2011;104:1071-1078.

6. Kim JH, Oh SY, Kwon HC, et al. Phase II study of gemcitabine plus cisplatin in patients with anthracycline- and taxane- pretreated metastatic breast cancer. Cancer Res Treat 2008;40:101-105.

7. Lee J, Kim HH, Ro SM, Yang JH. Capecitabine and cisplatin (XP) combination systemic chemotherapy in heavily pre-treated HER2 negative metastatic breast cancer. PLoS One 2017;12:e0171605.

8. Staudacher L, Cottu PH, Dieras V, et al. Platinum-based chemotherapy in metastatic triple-negative breast cancer: the Institut Curie experience. Ann Oncol 2011;22:848-856.

9. Shamseddine AI, Farhat FS. Platinum-based compounds for the treatment of metastatic breast cancer. Chemotherapy 2011;57:468-487.

10. Li Q, Li Q, Zhang P, et al. A phase II study of capecitabine plus cisplatin in metastatic triple-negative breast cancer patients pretreated with anthracyclines and taxanes. Cancer Biol Ther 2015;16:1746-1753.

11. Kohail H, Shehata S, Mansour O, et al. A phase 2 study of the combination of gemcitabine and cisplatin in patients with locally advanced or metastatic breast cancer previously treated with anthracyclines with/without taxanes. Hematol Oncol Stem Cell Ther 2012;5:42-48.

12. Park YH, Jung KH, Im SA, et al. Phase III, multicenter, randomized trial of maintenance chemotherapy versus observation in patients with metastatic breast cancer after achieving disease control with six cycles of gemcitabine plus paclitaxel as first-line chemotherapy: KCSGBRo7-02. J Clin Oncol 2013;31:1732-1739.

13. Paz-Ares LG, de Marinis F, Dediu M, et al. PARAMOUNT: final overall survival results of the phase III study of maintenance pemetrexed versus placebo immediately after induction treatment with pemetrexed plus cisplatin for advanced nonsquamous non-small-cell lung cancer. J Clin Oncol 2013;31:2895-2902.

14. Loibl S, Gianni L. HER2-positive breast cancer. Lancet 2017;389:2415-2429.

15. Gerber DE, Schiller JH. Maintenance chemotherapy for advanced non-small-cell lung cancer: new life for an old idea. J Clin Oncol 2013;31:1009-1020.

16. Seo JH, Oh SC, Choi CW, et al. Phase II study of a gemcitabine and cisplatin combination regimen in taxane resistant metastatic breast cancer. Cancer Chemother Pharmacol 2007;59:269-274.

17. Zhang J, Wang Z, Hu X, et al. Cisplatin and gemcitabine as the first line therapy in metastatic triple negative breast cancer. Int J Cancer 2015;136:204-211. 


\section{Supplementary Table 1. Patient characteristics}

\begin{tabular}{|c|c|c|}
\hline Characteristic & $\begin{array}{l}\text { Patients treated with cisplatin-doublet } \\
\text { regime }(n=96)\end{array}$ & $\begin{array}{l}\text { Chemotherapy holiday } \\
\qquad(\mathrm{n}=9)\end{array}$ \\
\hline Age, yr & $52(30-70)$ & $57(34-65)$ \\
\hline$<65$ & $90(93.8)$ & $8(88.9)$ \\
\hline$\geq 65$ & $6(6.2)$ & $1(11.1)$ \\
\hline \multicolumn{3}{|l|}{ ECOG } \\
\hline $0-1$ & $82(85.4)$ & $8(88.9)$ \\
\hline 2 & $14(14.6)$ & $1(11.1)$ \\
\hline \multicolumn{3}{|l|}{ Hormone receptor status } \\
\hline Positive & $66(68.8)$ & $7(77.8)$ \\
\hline Negative & $30(31.2)$ & $2(22.2)$ \\
\hline HER2 positive & $17(17.7)$ & $3(33.3)$ \\
\hline Triple negative & $21(21.9)$ & $1(11.1)$ \\
\hline \multicolumn{3}{|l|}{ Disease status } \\
\hline Metastatic breast cancer & $77(80.2)$ & $8(88.9)$ \\
\hline Recurrent breast cancer & $19(19.8)$ & $1(11.1)$ \\
\hline \multicolumn{3}{|l|}{ Neoadjuvant chemotherapy } \\
\hline Anthracycline & $14(14 \cdot 6)$ & $3(33 \cdot 3)$ \\
\hline Taxane & $7(7 \cdot 3)$ & $2(22.2)$ \\
\hline \multicolumn{3}{|l|}{ Adjuvant chemotherapy } \\
\hline Anthracycline & $48(50.0)$ & $4(44 \cdot 4)$ \\
\hline Taxane & $22(22.9)$ & $1(11.1)$ \\
\hline \multicolumn{3}{|l|}{ Previous lines of palliative chemotherapy } \\
\hline Naïve & $7(7 \cdot 3)$ & $1(11.1)$ \\
\hline 1st & $30(31.3)$ & $3(33.3)$ \\
\hline 2nd & $19(19.7)$ & $1(11.1)$ \\
\hline 3rd & $14(14.6)$ & o \\
\hline$\geq 4^{\text {th }}$ & $26(27.1)$ & $4(44.4)$ \\
\hline \multicolumn{3}{|l|}{ Previous chemotherapy agents } \\
\hline Gemcitabine & $8(8.3)$ & o \\
\hline Vinorelbine & $21(21.9)$ & $1(11.1)$ \\
\hline Eribulin & $3(3.1)$ & o \\
\hline CMF & $28(29.2)$ & $3(33 \cdot 3)$ \\
\hline Capecitabine & $22(22.9)$ & $8(88.9)$ \\
\hline Previous palliative endocrine treatment & $36(37.5)$ & $6(66.7)$ \\
\hline Previous palliative HER2 treatment & $13(13.5)$ & $2(22.2)$ \\
\hline \multicolumn{3}{|l|}{ No. of metastatic sites } \\
\hline 1 & $28(29.2)$ & $1(11.1)$ \\
\hline 2 & $27(28.1)$ & $1(11.1)$ \\
\hline$\geq 3$ & $41(42.7)$ & $7(77.8)$ \\
\hline \multicolumn{3}{|l|}{ Metastatic sites } \\
\hline Distant lymph nodes & $39(40.6)$ & $5(55.6)$ \\
\hline Lung & $40(41.7)$ & $5(55 \cdot 6)$ \\
\hline Liver & $29(30.2)$ & $1(11.1)$ \\
\hline Bone & $45(46.9)$ & $6(66.7)$ \\
\hline Pleura & $17(17.7)$ & $2(22.2)$ \\
\hline Brain & $15(15 \cdot 6)$ & $4(44 \cdot 4)$ \\
\hline Visceral metastases & $74(77.1)$ & $8(88.9)$ \\
\hline
\end{tabular}

Values are presented as median (range) or number (\%).

ECOG, Eastern Cooperative Oncology Group; HER2, human epidermal growth factor 2; CMF, cyclophosphamide (oral) + methotrexate +5 -fluorouracil. 
Joung EK, et al. Maintenance chemotherapy in MBC

Supplementary Table 2. Clinical outcomes during initial 6 cycles of cisplatin-doublet chemotherapy

\begin{tabular}{lcc}
\hline Variable & Gemcitabine cisplatin & Capecitabine cisplatin \\
\hline Best response & $15(15.6)$ & $21(21.9)$ \\
$\quad$ Partial response & $8(8.3)$ & $34(35.4)$ \\
$\quad$ Stable disease & $36(37.5)$ \\
Overall response rate & $78(81.3)$ \\
Disease control rate & $5.13(4.51-5.74)$ & $4.23(3.41-5.05)$ \\
Survival outcome & 5 \\
\hline
\end{tabular}

Values are presented as number (\%) or $95 \%$ confidence interval.

PFS, progression-free survival. 
Supplementary Table 3. Systemic treatment after progression

\begin{tabular}{|c|c|}
\hline Variable & Chemotherapy holiday $(\mathrm{n}=9)$ \\
\hline \multicolumn{2}{|c|}{$\begin{array}{l}\text { Subsequent chemotherapy } \\
\text { regimens }\end{array}$} \\
\hline Total & 17 \\
\hline 1 & 6 \\
\hline 2 & 4 \\
\hline$\geq 3$ & 7 \\
\hline Median & 3 \\
\hline No. of total cycles & 89 \\
\hline \multicolumn{2}{|l|}{$\begin{array}{l}\text { Type of chemotherapy } \\
\text { regimens }\end{array}$} \\
\hline Capecitabine & 5 \\
\hline \multicolumn{2}{|l|}{ Gemcitabine } \\
\hline Eribulin & 4 \\
\hline Vinorelbine & 1 \\
\hline $\mathrm{T}-\mathrm{DM} 1$ & 1 \\
\hline \multicolumn{2}{|l|}{ Paclitaxel } \\
\hline Docetaxel & 3 \\
\hline Nab-paclitaxel & 1 \\
\hline $\mathrm{CMF}$ & 2 \\
\hline \multicolumn{2}{|l|}{$\begin{array}{l}\text { Subsequent endocrine } \\
\text { regimens }\end{array}$} \\
\hline Total & 5 \\
\hline 1 & 2 \\
\hline 2 & 2 \\
\hline$\geq 3$ & 1 \\
\hline \multicolumn{2}{|l|}{ Type of endocrine therapy } \\
\hline \multicolumn{2}{|l|}{ Tamoxifen } \\
\hline Letrozole & 2 \\
\hline Exemestane & 1 \\
\hline Exemestane + everolimus & 1 \\
\hline Fulvestrant & 1 \\
\hline
\end{tabular}

$\overline{\mathrm{T}-\mathrm{DM} 1, \text { trastuzumab emtansine; } \mathrm{CMF} \text {, cyclophosphamide }}$ (oral) + methotrexate + 5-fluorouracil . 